\title{
IV.2
}

\section{EVIDENCE FOR $d$-WAVE ORDER PARAMETER SYMMETRY IN Bi-2212 FROM EXPERIMENTS ON INTERLAYER TUNNELING}

\author{
Yu I. Latyshev \\ Inst. of Radio-Engineering and Electronics, Russian Acad. of Sci., Mokhovaya 11-7, Moscow \\ 101999
}

\begin{abstract}
We consider three group experiments on interlayer tunneling on $\mathrm{Bi}-2212$ mesa-type structures, fabricated by focused ion beam (FIB) technique from Bi2212 single crystal whiskers, pointing out to the $d$-wave type of the order parameter (OP) in this compound. We specify the experiments on low temperature interlayer quasiparticle conductivity and magneto-conductivity on small Bi-2212 mesas and the experiments on Josephson flux-flow dissipation on long Bi-2212 mesas. All the results are shown to be consistent with a $d$ wave Fermi-liquid model with a significant contribution of coherent interlayer tunneling.
\end{abstract}
Key words: $\mathrm{Bi}_{2} \mathrm{Sr}_{2} \mathrm{CaCu}_{2} \mathrm{O}_{8+\mathrm{x}} c$-axis Transport and Magneto-transport, Single Crystal Whiskers, $d$-wave, Order Parameter Symmetry, Josephson Flux-flow Resistivity.

\section{INTRODUCTION}

More than 15 years studies of high temperature superconductivity in cuprates accumulated many evidences of the $d$-wave type symmetry of the superconducting order parameter (OP) in these materials. The most strong ones has been found from the ARPES experiments [1], quantum interference 
on tricrystal boundary [2,3], experiments on $\mathrm{YBCO} / \mathrm{Nb}$ zigzag junctions [4] etc. Here we consider several experiments on interlayer tunneling on Bi2212 mesa-type structures that also points out to the $d$-wave symmetry of the OP. The advantage and the complement of this method to other techniques is that that is essentially bulk method. The experiments considered below have been carried out on the $c$-axis structures fabricated from single crystal whiskers, the very perfect single crystal objects $[5,6]$.

The interlayer tunneling technique $[7,8]$ is based on the layered crystalline structure of the highly anisotropic high temperature superconductors as $\mathrm{Bi}-$ and Ta-based materials. Microscopically this structure represents a stack of elementary superconducting layers separated by elementary oxide layers that forms a series of elementary tunneling junctions. To study the interlayer Josephson tunneling correctly one needs to have a stack structure of the micron scale lateral size, $L, L<\lambda_{J}=s \lambda_{c} / \lambda_{a b}$, where $s$ is the spacing between elementary superconducting layers $(\mathrm{s}=15.6$ $\AA$ for Bi-2212) and $\lambda_{a b, c}$ is the anisotropic London penetration depth $\left(\lambda_{c} / \lambda_{a b} \approx\right.$ 500 for slightly overdoped Bi-2212), that contains also a small enough number of elementary junctions, $N$, typically $N$ being of few tens. In the opposite limit of long junctions $L>>\lambda_{J}$ the interlayer Josephson tunneling is essentially affected by the presence of Josephson vortices.

\section{FABRICATION OF THE C-AXIS STRUCTURES}

The Bi-2212 single crystal whiskers have been grown by impurity-free method [6]. Thin whiskers have been characterized as a very perfect single crystal objects. They grow along the $a$-axis free of any crucibles or substrates and can be entirely free of macroscopic defects and dislocations. They can reach in a length the size up to $10 \mathrm{~mm}$ having, however, the typical size below $1 \mathrm{~mm}$. In the $b c$-plane they have rectangular cross-section with typical sizes $L_{b}=1-30 \mu \mathrm{m}, L_{c}=0.1-3 \mu \mathrm{m}$. 


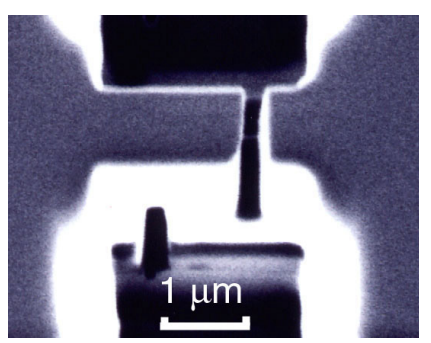

Figure IV:2:1. SEM picture of the typical Bi-2212 stacked structure, z-direction corresponds to the c-axis.

For the fabrication of stacked junctions we used focused ion beam technique. This technique has been developed for fabrication of both, the short [9] down to submicron scale [10] and the long junctions with a length of several tens microns [17]. For fabrication we used conventional FIB machine of Seiko Instr. Corp., SMI 9800 (SP) with Ga+-ion beam. The four leads were attached outside the junction area. The contact Ag pads were ablated and annealed before the FIB processing to avoid diffusion of $\mathrm{Ga}$-ions into the junction body. The example of a short stack fabricated by FIB technique is shown in Fig. 1. Typically we had slightly overdoped stacked $\mathrm{Bi}_{2} \mathrm{Sr}_{2} \mathrm{CaCu}_{2} \mathrm{O}_{8+\delta}$ structures with $\delta \approx 0.25$. They have $T_{c}=77 \mathrm{~K}, \rho_{c}(300 \mathrm{~K})=$ $10-12 \mathrm{Ohm} \mathrm{cm}, J_{c}(4.2 \mathrm{~K}) \approx 1 \mathrm{kA} / \mathrm{cm}^{2}$.

\section{THE c-AXIS TRANSPORT ON SHORT STACKS}

Here we consider results on short stacks with typical lateral size $1 \mu \mathrm{m} \times 1$ $\mu \mathrm{m}$ and containing $N=30-70$ elementary junctions. The high quality of the mesas has been approved by the Fraunhofer patterns of critical current $I_{c}$ on parallel magnetic field with periodicity of one flux per elementary junction $[11,12]$. Fig 2 shows the $I-V$ characteristics of the short stacked junctions in large and small voltage scales. 

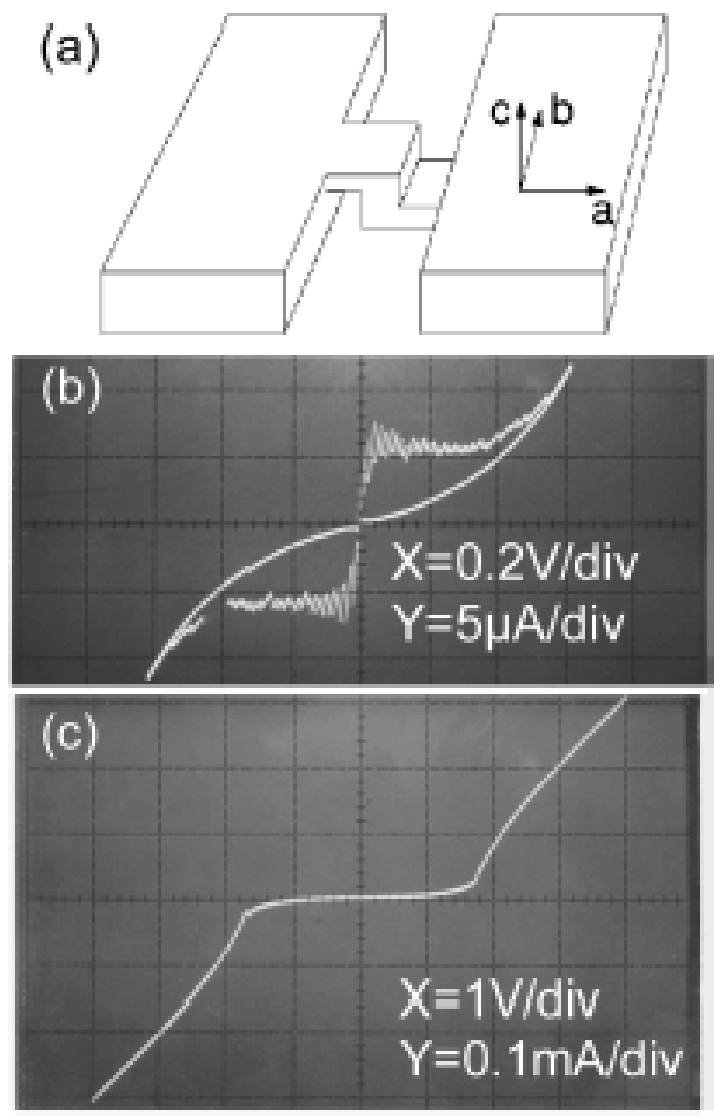

Figure IV:2:2. Schematic view of the junction (a); and the I-V characteristics of the Bi-2212 stacks in (b) enlarged scale for sample \#3 [14] and (c) extended scale for sample \#4 [12]. T = $4.2 \mathrm{~K}$

The superconducting gap voltage, $V_{g}$, (Fig.2c) was determined as the voltage of the maximum of the $d I / d V(V)$. The gap of intrinsic junction $2 \Delta_{0} \approx$ $e V_{g} / N$ reaches value as high as $50 \mathrm{meV}$ [10]. The multibranched structure (Fig.2b) corresponds to subsequent transition of the intrinsic junctions into the resistive state for increasing voltage [7]. At voltages $V>V_{g}$ all junctions are resistive. Therefore in downsweep of voltage, starting from $V>V g$, the $I$ $V$ curve is observed in the all junction resistive state. Here only 
quasiparticles contribute to the $c$-axis transport. Corresponding quasiparticle conductivity, $\sigma_{q}$, thus can be defined directly from that part of the $I-V$ curve.

The Ohmic resistance, $R_{n}$, at $V>V_{g}$ is also well defined from the $I-V$ characteristics (Fig. 2c). This resistance is nearly temperature independent and corresponds to the conductivity $\sigma_{n}\left(V>V_{g}\right) \approx 80(\mathrm{kOhm} \mathrm{cm})^{-1}$ for energies above superconducting gap. The critical current $I_{c}$ was determined from the $I-V$ characteristics as the current of switching from the superconducting to the resistive state, averaged over the stack. The variation of the critical current along the stack is not large (usually within 15\%), indicating a good uniformity of our structures. The $c$-axis critical current density was typically $\sim 1 \mathrm{kA} / \mathrm{cm}^{2}[12,13]$.

We found out that interlayer tunneling $I-V$ characteristics at low temperatures essentially differ from those of conventional Josephson junctions between s-wave superconductors. We specify [14]: 1) $J_{c}(0)$ is strongly reduced to compare with the value expected from AmbegaokarBaratoff (A-B) relation, $\left.J_{c}^{A B}(0)=\pi \sigma_{n} \Delta_{0} / 2 e s ; 2\right)$ quadratic and scaling behaviour of $\sigma_{q}(V, T)$ on $V$ and T (Figs. 3,4):

$$
\sigma_{q}(V, T)=\sigma_{q}(0,0)\left\{\begin{array}{l}
\left(1+b V^{2}\right) \\
\left(1+c T^{2}\right)
\end{array}\right.
$$

with $b=0.014 \pm 0.03 \mathrm{mV}^{-2}, c=(6 \pm 2) \times 10^{-4} \mathrm{~K}^{-2}$ and $c / b \sim 4$; 3$)$ nonzero and universal value of $\sigma_{q}(0,0) \approx 2.5(\mathrm{kOhm} \mathrm{cm})^{-1}$.

We found empirically the modified relation of the A-B type between $J_{c}$ and $\sigma_{q}$ :

$$
J_{c} \approx \pi \sigma_{q}(0,0) \Delta_{0} / 2 e s
$$

It was shown then that all these observed features can be described selfconsistently by Fermi-liquid model for quasiparticles in clean $d$-wave superconductor with resonant intralayer scattering [14]. The superconducting gap is expressed as $\Delta(\varphi)=\Delta_{0} \cos 2 \varphi$, where $\varphi$ is the angle of the momentum on the two-dimensional cylindrical Fermi-surface. 


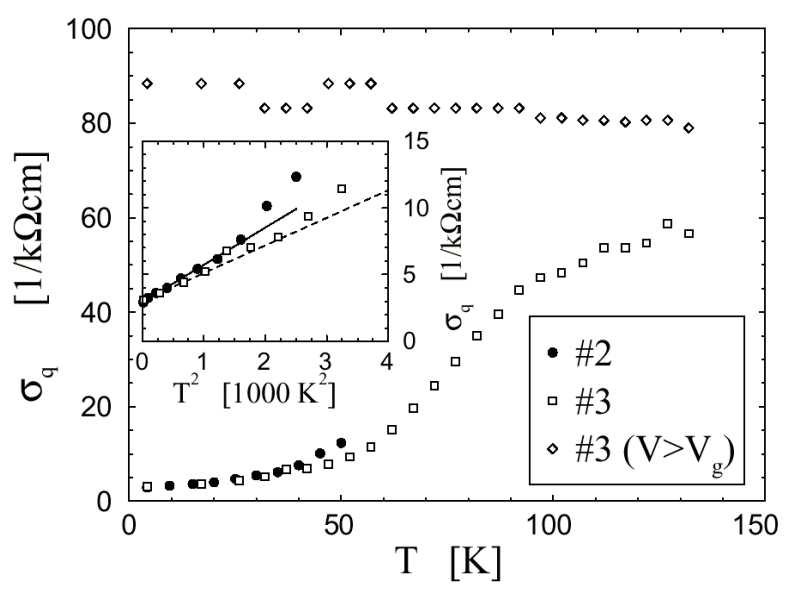

Figure IV:2:3 Quasiparticle dynamic conductivity $\sigma_{c}$ vs $T$ for voltages $v>V_{g} / N \approx 2 \Delta_{0} / e$ and $v$ $=V / N \rightarrow 0$ as extended from the $I-V$ characteristics of samples \#2 and \#3 [14]. The insert shows $\sigma_{c}$ vs $T^{2}$ at $v \rightarrow 0$. Lines are fit for $T^{2}<1000 \mathrm{~K}^{2}$.

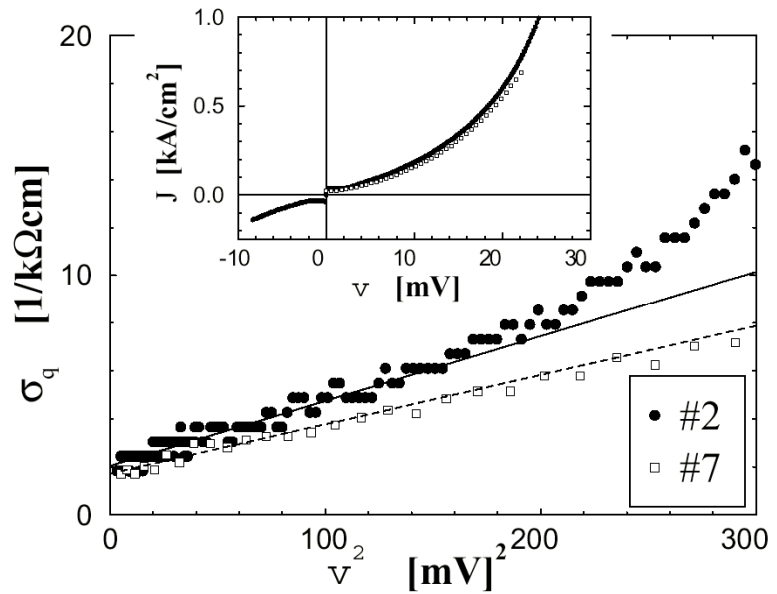

Figure IV:2:4. The quasipartical differential conductivity $v^{2}=V^{2} / N^{2}$ at $\mathrm{T}=4.2 \mathrm{~K}$ as extracted from the $I-V$ characteristics of sample $\# 2$ and \#7 (Fig. 4c in Ref. [8]). Lines are fits for $v<10 \mathrm{mV}$. Insert: Corresponding $J-v$ curves. 
Impurity scattering leads to the formation of the gapless state at some sectors $\varphi_{0}$ near the node directions $\varphi_{g}$ at angles $\varphi_{g} \pm \varphi_{0} / 2$, with $\varphi_{0} \sim \gamma / \Delta_{0}$, where $\gamma$ is the impurity bandwidth of quasiparticles. That results in a nonzero density of states at zero energy, $N(0)$, where $N(0)$ is the $2 \mathrm{D}$ density of states per spin at the Fermi level, and leads to a universal quasiparticle interlayer conductivity $\sigma_{q}(0,0)$ :

$$
\sigma_{q}(0,0) \propto \frac{N(0) \varphi_{0}}{\gamma}=\frac{N(0)}{\Delta_{0}}
$$

As that follows from Eq. (3), $\sigma_{q}(0,0)$ is independent on impurity scattering rate $\gamma$. The expression (3) is valid for the coherent interlayer tunneling that conserves the in-plane momentum in tunneling process. In more general case one can introduce coherence factor characterizing a weight of coherent tunneling as $a$ while the weight of incoherent one as $(1-a)$. Then one can get the following expressions for $J_{c}(0), \sigma_{q}(0,0), b$ and $c$ [14]:

$$
\sigma_{q}(0,0)=\frac{2 e^{2} t_{\perp}^{2} N(0) s}{\pi \hbar \Delta_{0}}\left[a+(1-a) \frac{\gamma}{\varepsilon_{F}}\right] ; \quad J_{c}(0)=\frac{e t_{\perp}^{2} N(0)}{\hbar}\left[a+(1-a) \frac{\Delta_{0}}{\varepsilon_{F}}\right]
$$

This theory also gives the scaling behaviour of $\sigma_{q}(V, T)$ of the type of Eq. (1):

$$
b=\frac{1}{8 \gamma^{2}}\left[1+\frac{(1-a)}{a} \frac{\gamma}{\varepsilon_{F}}\right] ; c=\frac{\pi^{2}}{18 \gamma^{2}}\left[1+\frac{(1-a) \gamma}{\varepsilon_{F}}\right],
$$

where $t_{\perp}$ is a tunneling matrix element [14]. One can see that the ratio $J_{c}(0) / \sigma_{q}(0,0)$ from Eq. (4) turns to the experimentally found expression (2) only for significant contribution of coherent tunneling, $a>>\max \left\{\Delta_{0} / \varepsilon_{F}\right.$, $\left.\gamma / \varepsilon_{F}\right\}$. From experimental value for $c$ we can estimate $\gamma$ using Eq. (5) as to be $\gamma \approx 3 \mathrm{mV}$. Then for $\Delta_{0} / \varepsilon_{F} \approx 0.1 \mathrm{we}$ can get estimation for $a, a>>0.1$. In that case $c / b=4 \pi^{2} / 9 \approx 4.4$ in a good agreement with experiment. Also, for $\sigma_{q}(0,0)$ we can get from Eq. (4), using $J_{c}=0.8 \mathrm{kA} / \mathrm{cm}^{2}, \Delta_{0}=30 \mathrm{meV}, s=1.5$ $10^{-7} \mathrm{~cm}$, the value $2.6(\mathrm{kOhm} \mathrm{cm})^{-1}$ which is very close to the experiment. 
Thus we can infer that experimental low $V, T$ interlayer tunneling data are consistent with Fermi-liquid picture taking into account $d$-wave symmetry and essentially coherent interlayer tunneling.

4. THE C-AXIS MAGNETO-CONDUCTANCE IN BI 2212

In this part we consider the $c$-axis magneto-transport on single crystals and small mesas in high magnetic field perpendicular to the layers. In both cases we access qasiparticle current by suppressing Josephson interlayer current in one of two ways: (a) by magnetic field of 60T in a case of single crystals, (b) by current that driving a small mesa in the all junction resistive state, as that was discussed above.

The $c$-axis magneto-resistance of Bi-2212 single crystals at different temperatures is shown in Fig. 5.

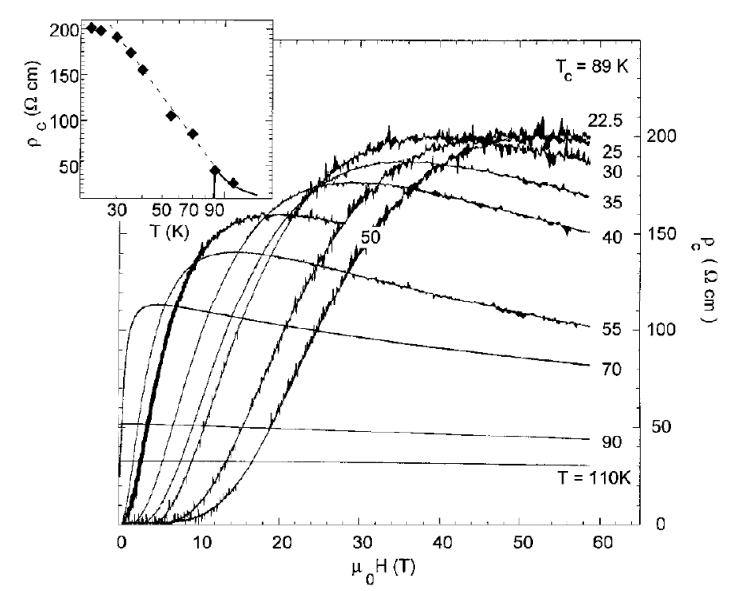

Figure $I V: 2: 5$. The out-of-plane resistivity $\rho_{c}$ vs magnetic field of a slightly overdoped Bi2212 crystal in fields up to $60 \mathrm{~T}$ at different temperat ures [15]. $J=0.05 \mathrm{~A} / \mathrm{cm}^{2}$. Insert: $\rho_{c}$ vs $T$ at $55 \mathrm{~T}$ (full rhombus) and $0 \mathrm{~T}$ (line).

The characteristic feature is that magneto-resistance achieves a maximum at some field and then drops down demonstrating negative magneto-resistance. 
Fig.6 shows the data of magneto-conductivity versus magnetic field partly including the data of Fig.5. This graph shows two contributions to magnetoconductivity $\sigma_{c}(H, T)[15]$ : (1) contribution of Josephson tunneling $\sigma_{J}$ which drops rapidly with field as $\sigma_{J}=\alpha H^{v}$, where the power index $v$ varies from 1.5 to 3.5 with temperature variation from $70 \mathrm{~K}$ to $22.5 \mathrm{~K}$; (2) contribution of quasiparticles $\sigma_{q}(H, T)=\sigma(0, T)[\eta+\beta H]$, where $\sigma_{q}(0, T)=\sigma_{q}(0,0)(1+$ $\left.c T^{2}\right)$ with $\sigma_{q}(0,0)=2.5(\mathrm{kOhm} \mathrm{cm})^{-1}$ as that was discussed before [14]. Both contributions plotted for $\mathrm{T}=55 \mathrm{~K}$ at Fig. 6 quite well describe experimental dependence of $\sigma_{q}(H, T)$ [15]. As shown at high enough field $\sigma_{J}(H)$ becomes negligibly small and the linear growth of $\sigma_{q}(H)$ or negative magnetoresistance is completely defined by quasiparticle contribution. That conclusion has been confirmed by studies of magneto-resistance on mesas in all junctions resistive state, Fig. 7. Thus experiment shows a linear growth of quasiparticle conductivity with field $\sigma_{q}(H, T)-\sigma_{q}(0, T) \propto \sigma_{q}(0,0) H$. This unusual behaviour has been explained in a $d$-wave Fermi-liquid model [16]. It was shown that at high field limit the variation of quasiparticle conductivity, $\Delta \sigma$, is expressed as follows [16], $\Delta \sigma_{q}(H) \sim \sigma_{q}(0,0) H / H_{\Delta}$, where $H_{\Delta}=\Phi_{0} \Delta_{0} / \hbar^{2} v_{F}^{2} \approx 40-80 \mathrm{~T}$. This dependence is consistent with experiment [15] (see Figs. 6,7)

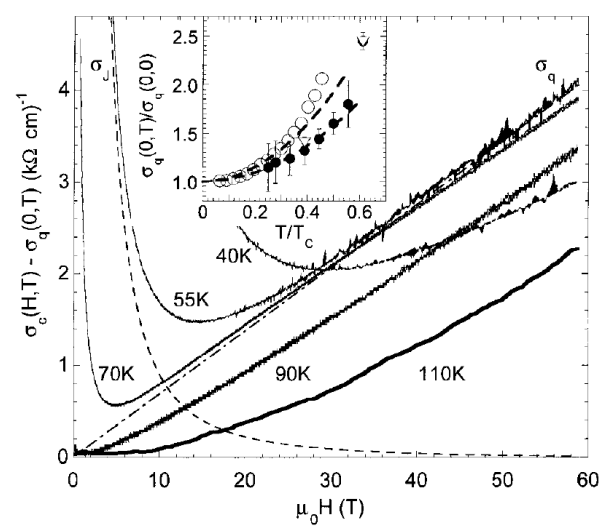

Figure IV:2:6. $\sigma_{c}(H)$ below and above $T_{c}=89 \mathrm{~K}$. A fit at $55 \mathrm{~K}$ to a superposition of Cooper pair (dashed line) and quasiparticle (dash-dotted line) contributions to magneto-conductivity is indicated (see text). Insert: Zero field $\sigma_{c}$ vs $T / T_{c}$ extracted from $\sigma_{c}(H, T)$ for a $\mathrm{Bi}-2212$ crystal for $J=0.05 \mathrm{~A} / \mathrm{cm}^{2}$ (full circles) and $J=0.1 \mathrm{~A} / \mathrm{cm}^{2}$ (triangles), and obtained from the $I-V$ curves for a mesa (empty circles). Both fit a $T^{2}$ dependence (dashed line) up to $T=\gamma$. 


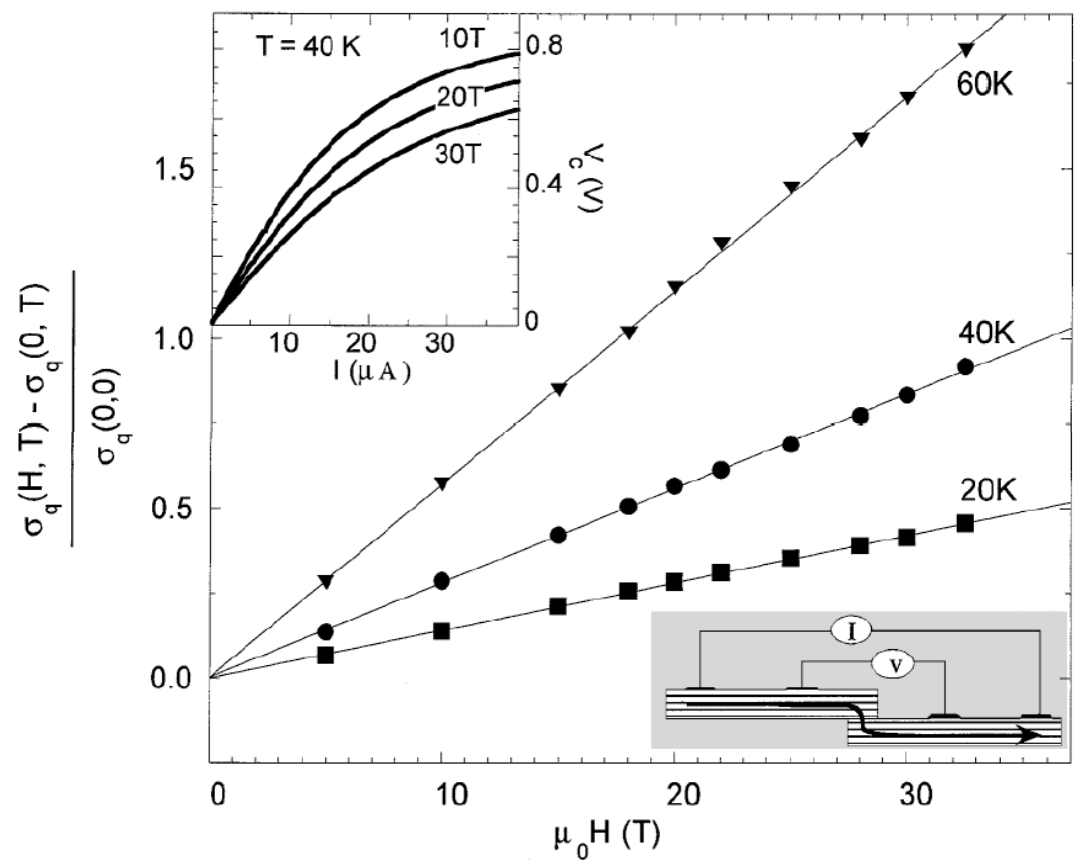

Figure IV:2:7. Normalized quasiparticle $c$-axis conductivity as a function of $H / / c$ obtained from the $I-V$ curves (top inset) measured on the mesa-shaped Bi-2212 (sketched) [15].

\section{JOSEPHSON FLUX-FLOW IN-PLANE DISSIPATION IN Bi-2212}

In this part we describe our experiments on studies of dissipation in Josephson flux-flow regime. We show that experiments of that type can be used for studies of temperature dependence of both the in-plane and the outof-plane components of the quasiparticle conductivity [17]. 

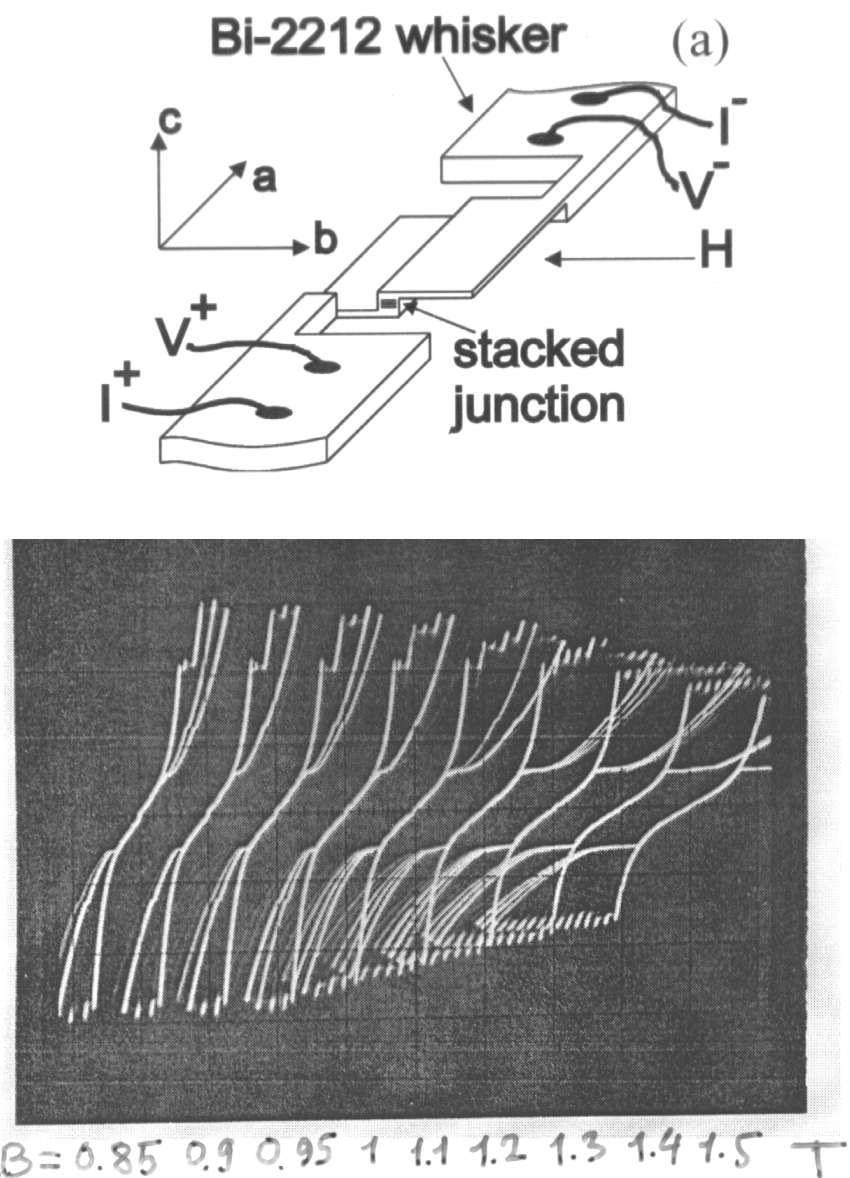

Figure $I V: 2: 8$. The set up (top insert) and the set of the $I-V$ characteristics measured in the flux-flow regime with field $B$ applied along the b-axis, $B$ increasing from $0.85 \mathrm{~T}$ up to $1.5 \mathrm{~T}$.

The unusual temperature dependence of $\sigma_{a b}(T)$ found from our experiment [17] is consistent with the microwave data [18] and can be 

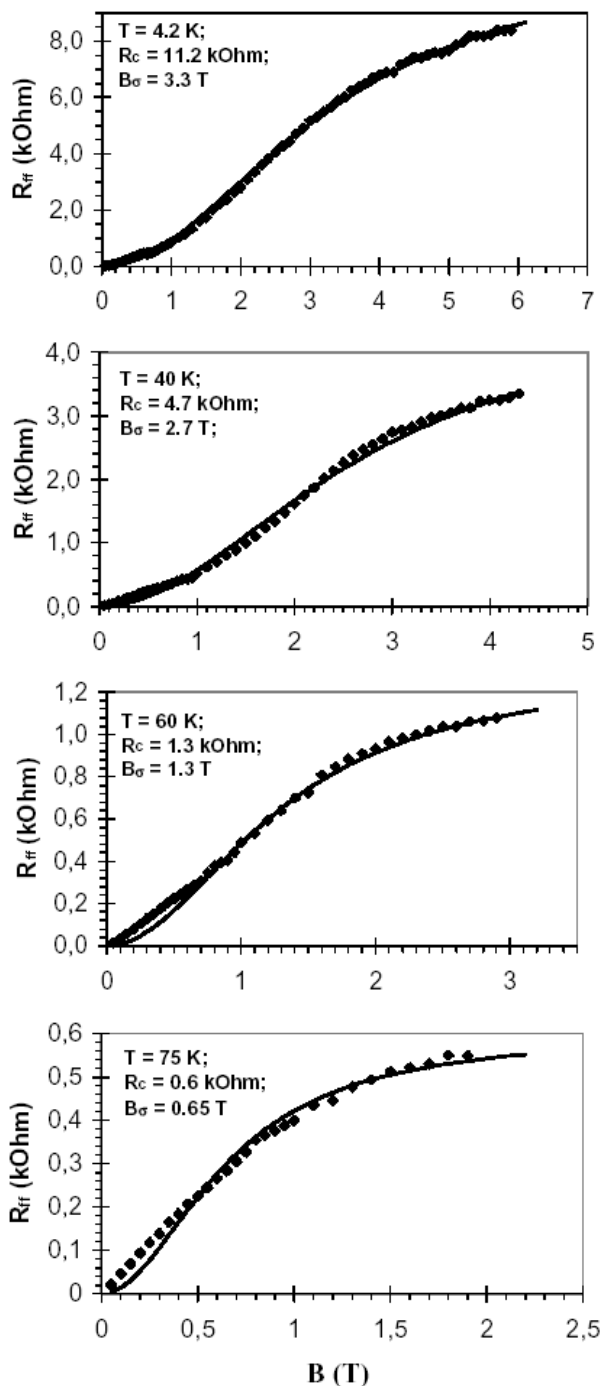

Figure IV:2:9. Magnetic field dependence of the Josephson flux-flow resistance, $R_{f f}$, at different temperatures with fits to Eq. (7) [17].

naturally explained by a $d$-wave Fermi-iquid model. As that is well known, magnetic field applied parallel to the layers of the layered high- $\mathrm{T}_{\mathrm{c}}$ superconductor like Bi-2212 (below we consider a long enough mesa- 
structure sketched at Fig.8) can introduce the Josephson vortices (JVs) [19] with characteristic sizes $\lambda_{J}$ along the layers and $\lambda_{a b}$ across the layers. They are centered between superconducting layers and have no normal core. A concentration of Josephson vortices grows with field and the interaction between them leads to formation of triangular Josephson vortex lattice (JVL) [20]. With field growth at $H>H_{0}=\Phi_{0} / \lambda_{J} S$ non-linear cores of Josephson vortices in one line start to overlap and so called dense JVL is formed. JVs strongly interact with each other in a dense JVL. As a consequence, when a DC current is applied across the layers, the resulting Lorentz force can drive a dense JVL as a whole [21]. This regime is known as Josephson flux-flow (JFF). That is characterized by a specific JFF branch on the IV characteristic having non-linear upturn with maximum voltage being proportional to the applied magnetic field (Fig. 8) [22]. In a linear JFF regime the dissipation is proportional to the linear slope of JFF branch at $\mathrm{V} \rightarrow 0$ (Fig. 8).In a general case that can be expressed as follows [23]:

$$
\sigma_{J F F} E_{z}^{2}=\sigma_{c} E_{z}^{2}+\sigma_{c} E_{z \omega}^{2}+\sigma_{a b} E_{x \omega}^{2} .
$$

Here directions $z$ and $x$ correspond to the orientation of electric field across and along the layers correspondingly, $E_{x, z}$ is the DC components of electric field, index $\omega$ is related to the AC electric field components associated with the motion of JVL. In earlier calculations of dissipation in JFF regime [19, 24] only two first terms of Eq. (6) have been taken into account. However, as that has been pointed out recently [23], the third term can have the leading contribution for the highly anisotropic layered superconductors, satisfying the condition $\Gamma=\left(\sigma_{a b} / \sigma_{c}\right) /\left(\lambda_{c}^{2} / \lambda_{a b}^{2}\right)>1$. For materials like BSCCO at low temperatures $\Gamma$ is typically about 50 . In this case the JFF resistivity was predicted to have unusual quadratic dependence on parallel magnetic field $\mathrm{B}$ [23]:

$$
\rho_{J f f}=\frac{B^{2}}{B^{2}+B_{\sigma}^{2}} \rho_{c}, \quad B_{\sigma}=\sqrt{\frac{\sigma_{a b}}{\sigma_{c}}} \frac{\Phi_{0}}{\sqrt{2}} \frac{1}{\pi \gamma^{2} s^{2}}
$$

The experiment shows very nice fit of $\rho_{J F F}(B)$ dependence to this expression [17] at wide temperature range, as shown at Fig. 9. 
That allows us to extract from the JFF experiment both components of quasiparticle conductivity $\sigma_{c}$ and $\sigma_{a b}$ and their temperature dependences [17] (Fig.10). As shown, they both are consistent with those measured by other independent methods.

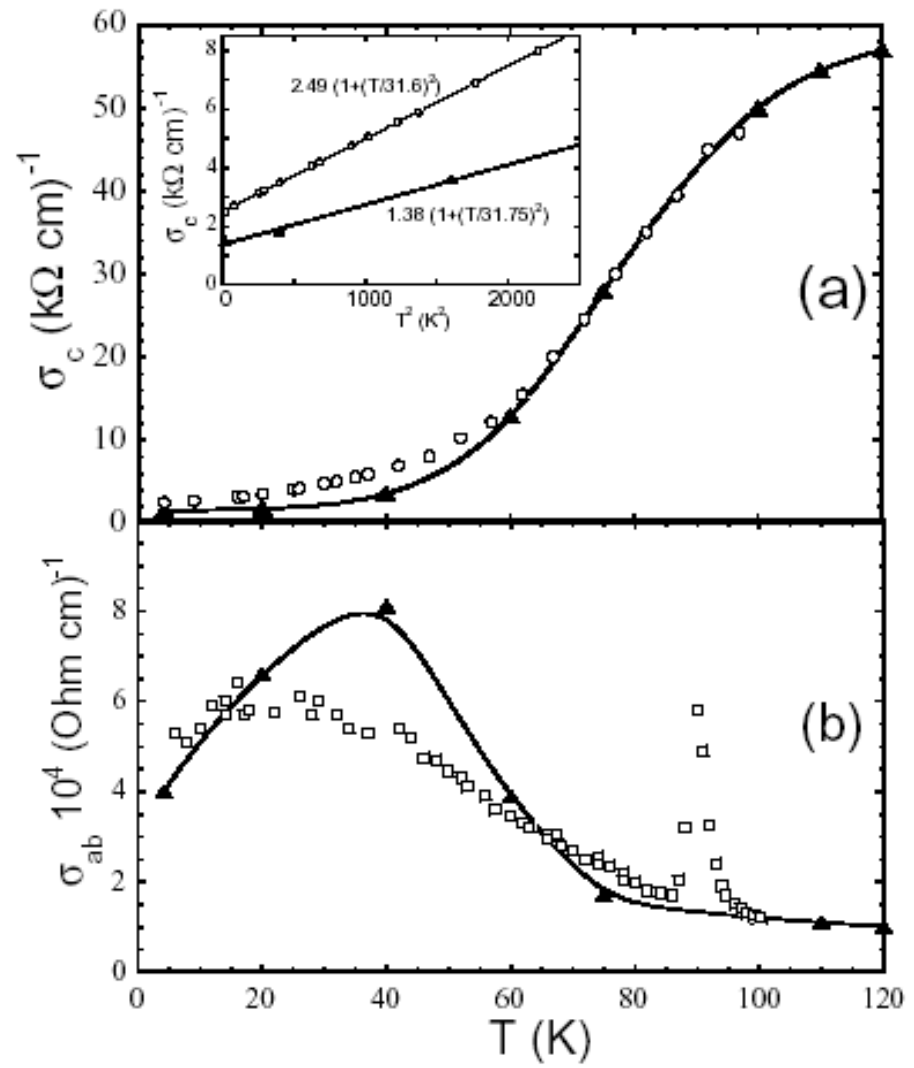

Figure IV:2:10. Solid triangles show temperature dependence of the out-of-plane quasiparticle conductivity $\sigma_{c}$ (a) and in-plane quasiparticle conductivity $\sigma_{a b}$ (b). Below $T_{c}$ they are extracted from the JFF experiment on BSCCO long stack [17] and above $T_{c}$ they represent the normal state conductivities of whiskers measured independently on samples from the same batch. Open circles correspond to the $\sigma_{\mathrm{c}}$ data from Ref.14, obtained on small mesas in zero field, open squares correspond to $14.4 \mathrm{GHz}$ microwave data for $\sigma_{a b}$ from Ref. 18 obtained on epitaxial films. Solid lines in both plots are just guides for the eye. Insert in (a) shows the low temperature part of $\sigma_{c}(T)$ plotted versus $T^{2}$. 
The most important result is that temperature dependence of the in-plane quasiparticle conductivity, $\sigma_{a b}(T)$, well reproduces unusual maximum at $T=$ $20-30 \mathrm{~K}$. This type of $\sigma_{a b}(T)$ behaviour has been found earlier in the microwave experiments for YBCO [25] and BSCCO [18] and also is consistent with the heat transport measurements of the electronic part of the thermal conductivity [26].

Fig.10b shows a comparison of our data with the microwave results [18]. The origin of the peak of $\sigma_{a b}(T)$ has been widely discussed as a result of a $d$ wave symmetry of the OP in BSCCO and YBCO. In particular, in a $d$-wave Fermi-liquid model it was shown that at low temperatures $\sigma_{a b}$ grows with temperature as [27]: $\sigma(\omega \rightarrow 0, T)=\sigma_{00}\left(1+\gamma T^{2}\right)$, where $\sigma_{00}$ is a universal inplane conductivity introduced by Lee [28], $\sigma_{00}=n e^{2} /\left(\pi m_{a b} \Delta_{0}\right)$ with $m_{a b}$ the effective quasiparticle in-plane mass.

Actually this growth is caused by thermally activation of nodal quasiparticles. At higher temperatures the quasiparticle relaxation time drops down [29]. The peak in a temperature dependence of $\sigma_{a b}$ appears then as a result of an interplay between the temperature dependences of the relaxation rate and concentration of quasiparticles.

\section{CONCLUSIONS}

The experiments on interlayer tunneling on short and long Bi-2212 mesas show new evidences of the $d$-wave OP symmetry. That results in: (1) the highly reduced value of the $c$-axis critical current density in comparison with the expected value from conventional Ambegaokar-Baratoff relation, (2) finite and universal value for the $c$-axis low temperature quasiparticle conductivity and its scaling relations on the bias voltage and temperature, (3) linear growth of magneto-conductivity in high magnetic fields, (4) the unconventional temperature dependence of the in-plane conductivity having a peak at $20-30 \mathrm{~K}$. 


\section{AKCNOWLEDGEMENTS}

We acknowledge support from the CRDF grant No RP1-12397-MO-02, grant from Russian Ministry of Science and Industry No. 40.012.1.111.46 and Jumelage project between IRE RAS and CRTBT CNRS,

No. 03-02-2201.

\section{REFERENCES}

1. Z-X. Shen et al. Phys. Rev. Lett. 70, 1553 (1993); Z-X. Shen et al., Science, 267, 343 (19995).

2. C.C. Tsuei et al. Phys. Rev. Lett. 73, 593 (1994).

3. J.R. Kirtley et al. Nature, 373, 225 (1995); C.C. Tsuei and J.R. Kirtley, Rev. Mod. Phys., 72, 969 (2000).

4. H.J.H. Smilde et al. Phys. Rev. Lett., 88, 05704 (2002).

5. I. Matzubara, H. Kageyama, T.Ogura, H. Yamashita, and T. Kawai, Jpn. J. Appl. Phys. 28, L 1121 (1989).

6. Yu.I. Latyshev, I.G. Gorlova, A.M. Nikitina, V.U. Antokhina, S.G. Zybtsev, N.P. Kukhta and V.N. Timofeev, Physica C 216, 471 (1993).

7. R. Kleiner, F.Steinmeyer, G. Kunkel, P.Muller, Phys. Rev. Lett., 68, 239, (1992); R.Kleiner and P. Mueller, Phys. Rev. B 49, 1327 (1994).

8. K. Tanabe, Y. Hidaka, S. Karimoto, M. Suzuki, Phys. Rev. B 53, 9348 (1996).

9. Yu.I. Latyshev, S.-J. Kim, T. Yamashita, IEEE Trans. Appl. Supercond., 9, 4312 (1999); S.-J. Kim, Yu.I. Latyshev and T. Yamashita, Supercond. Sci. Technol. 12, 728 (1999).

10. Yu.I. Latyshev, S.-J. Kim, T. Yamashita, JETP Lett., 69, 84 (1999).

11. L.N. Bulaevskii, J.R. Clem, L.I. Glazman, Phys. Rev. B 46, 350 (1992).

12. Yu.I. Latyshev, S.-J. Kim, V.N. Pavlenko, T. Yamashita, L.N. Bulaevskii, Physica C, 362, 156 (2001).

13. K. Inomata, T. Kawae, K. Nakajima, S.-J. Kim, and T. Yamashita, Appl. Phys. Lett. 82, 769 (2003).

14. Yu.I. Latyshev, T. Yamashita, L.N. Bulaevskii, M.J. Graf, A.V. Balatsky, and M.P. Maley, Phys. Rev. Lett. 82, 5345 (1999).

15. N. Morozov, L. Krusin-Elbaum, T. Shibauchi, L.N. Bulaevskii, M.P. Maley, Yu.I.Latyshev, and T.Yamashita, Phys. Rev. Lett. 84, 1784 (2000).

16. I. Vekhter, L.N. Bulaevskii, A.E. Koshelev, and M.P. Maley, cond-mat/9910341.

17. Yu.I. Latyshev, A.E. Koshelev, L.N. Bulaevskii, Phys.Rev. B 68, 134504 (2003).

18. Shin-Fu Lee et al., Phys. Rev. Lett., 77, 735 (1996); H. Kitano et al., J. Low Temp. Phys., 117, 1241 (1999).

19. J. Clemm and M. Coffey, Phys. Rev.B 42, 6209 (1990).

20. L.N. Bulaevskii and J.R. Clem, Phys. Rev., B 44, 10234 (1991). 
21. L.N. Bulaevskii, D. Domingez, M.P. Maley, A.R. Bishop, and B.I. Ivlev, Phys. Rev. B 53, 14601 (1996).

22. J.U. Lee et al., Appl. Phys. Lett., 67, 1471 (1995); G. Hechtfischer et al. Phys. Rev. Lett., 79, 1365 (1997); Yu.I. Latyshev et al., Physica C 293, 174 (1997).

23. A.E. Koshelev, Phys. Rev., B 62, R3616 (2000).

24. S. Sakai et al. Phys. Rev., B 50, 12905 (1994); M. Machida et al. Physica C, 330, 85 (2000).

25. D.A. Bonn et al., Phys. Rev. Lett., 68, 2390 (1992); D.A. Bonn et al., Phys. Rev. B 50, 4051 (19994); A. Hosseini et al., ibid. 60, 1349 (1999).

26. K. Krishna et al. Phys. Rev. Lett., 75, 3529 (1995); B. Zeini et al. Eur. Phys. J. B 20, 189 (2001).

27. P. J. Hirschfeld, W.O. Puttika, D.J. Scalapino Phys. Rev. B 50, 10250 (1994).

28. P.A. Lee, Phys.Rev. Lett, 71, 1887 (1993). 
